\title{
Three-Dimensional Micro Actuator and Sensor System for Dynamic Stimulations and Evaluations of Mechanical Properties of Minute Living Cells*
}

\author{
Ken-ichi KONNO $^{* *}$ Tadashi KOSAWADA ${ }^{* *}$ and Makoto AKUTSU ${ }^{* * *}$ \\ **Yamagata University, \\ 4-3-16 Jonan, Yonezawa, Yamagata 992-8510, Japan \\ E-mail: kosawada@yz.yamagata-u.ac.jp \\ ${ }^{* * \star}$ Fujirebio Inc. \\ 2-62-5 Nihonbashi Hamacho, Chuo-ku, Tokyo 103-0007, Japan
}

\begin{abstract}
In this study, a new noninvasive sensor system is developed to detect compliance of minute living cells and tissues by using dynamic response of a piezoelectric vibrator. The bending mode of vibration, excited impulsively by piezoelectric ceramics, is utilized in a small clamped-free beam type vibrator. A three-dimensional micro actuator, in which a doubly L-shaped clamped-free beam type vibrator is utilized, is also developed to enforce dynamic stimulations. The sensor and the three-dimensional actuator were united and integrally controlled by a micromanipulator system, while dimensions and morphologies of the cells were measured by an inverted phase contrast microscope system. Experimental studies have been carried out using fertilized egg cell of killifish. The studies have shown the sensor's capability to detect changes of mechanical properties of the minute living cells. Also, the method has shown that the reaction of living cells might have frequency dependence on vibrating stimulations by the actuator.
\end{abstract}

Key words: Three Dimensional Micro Actuator, Micro Sensor, Piezoelectric Device, Dynamic Stimulation, Living Cell, Mechanical Property, Locally Damaged Cell, Reaction of Cell

\section{Introduction}

Sensing systems and criteria for normal industrial materials and objects have well been established. However, these are not well developed especially in case of very soft materials or living objects whose sizes are in micron meter order or less. This is mainly because of difficulties in sensing and holding architecture as well as in sensitive manipulation of these objects. Furthermore, noninvasive or low invasive sensing procedures are desirable or sometimes indispensable for the most living objects. If one can develop a reliable method to detect mechanical properties of the minute soft materials or living objects, it will provide us significant information not only as a means of diagnosis in medical examinations like softness of skins, internal organs and even cells but also as a standard for design of various sensors and instruments used in medical, engineering and scientific purposes.

The cell is a minimum unit of life and its structure-function relationship ${ }^{(1)}$ is of fundamental importance both in science and biomedical engineering. When the cell is under stress stimulations from outside such as changes of temperature, concentration and

*Received 28 Apr., 2006 (No. 06-0022) [DOI: 10.1299/jbse.1.147] 
$\mathrm{pH}$ of its surrounding media, the inside structure of the cell makes changes in order to initiate functions of defense, adaptation or transport. These functional adjustments cause changes in mechanical properties ${ }^{(2)}$ such as an increase of surface tension, stiffening, deformation and locomotion, some of which are considered as the target of measurements by the present sensing system. On the other hand, it is commonly pointed out that appropriate dynamic stimulations can initiate potential regeneration capability of the cell and lead to recovery from local damage ${ }^{(3)}$. However, the method has not developed yet due to difficulties not only in biology and medicine but also in engineering aspects.

So far, only limited studies have been reported in this field. Omata ${ }^{(4)}$ has developed a micro tactile sensor to detect softness of cells using longitudinal vibrations of a rod shaped piezoelectric element. Since the vibrations are only exited in longitudinal direction, the natural frequency of the sensor is high such as $200 \mathrm{kHz}$ and the softness in the vertical direction to the sensor probe is mainly given. Kosawada and Kuni- ${ }^{(5)}$, Kosawada et al. ${ }^{(6)(7)}$ have presented a sensing system utilizing bending mode of vibration of a small free-free beam type vibrator, whose natural frequency is comparatively low such as less than $10 \mathrm{kHz}$ and is easy to handle by a conventional FFT analyzer. The softness in the horizontal direction to the sensor probe is mainly given. However, free-free boundary condition of the vibrator is effective only for very soft object with small sensor indentation. Recently, Kosawada et al ${ }^{(8)}$ presented an improved sensor system with a clamped-free beam type vibrator. It has a stable and wide sensing range in detecting environmental stresses induced in living cells and tissues. These studies have mainly focused on sensing capabilities and do not have actuating capabilities to actively enforce the dynamic stimulations upon living cells.

Recently, an in-vitro fertilization technique including preimplantation genetic diagnosis as well as sex selection by obtaining a sample from an egg cell has been well developed in medical and stock breeding community. On the other hand, a method has not yet been developed to accelerate recovery process of the locally damaged egg cell and that to raise its survival rate. Therefore, it is much needed to develop an effective technique to foster the cell's physiological ability for regeneration.

In this study, we developed a new small sized three-dimensional actuator to enforce dynamic stimulations upon living cells. Incorporating the clamped-free sensor mentioned above into this three-dimensional actuator, we developed a three-dimensional actuator-sensor complex system, which enable us to have stimulation and detection onto the living cell in almost simultaneously. As a first example, the physiological responses to chemically environmental stress of fertilized egg cells of killifish were examined. Secondly, mechanical reactions of the cells against dynamic stimulations were investigated. Finally, we examined the recovery progress of the locally damaged cells by using the developed actuator-sensor complex system.

\section{Methods of experiments}

\subsection{Experimental setup and its improvements}

When vibrating end of a vibrator is in contact with a soft object, the natural frequency of the vibrator changes according to its dynamic constraints at the end boundary. This frequency change is related to the mechanical properties of the soft object. It is the compliance due to movability at contact point or vibration transmissibility of its surrounding area. Based on this concept, a noninvasive micro sensor as well as a three-dimensional micro actuator is developed as follows. Construction of the three-dimensional actuator-sensor complex is shown in Fig. 1(a). The second mode of vibration of a beam vibrator with clamped-free boundary conditions is utilized for the sensor because of its stability and large vibration displacement at the vibrating free end. In 
order to excite the second mode of vibration, a $20.0 \times 3.0 \times 0.2 \mathrm{~mm}$ stainless-steel plate was used for the vibrator. Two pieces of piezoelectric ceramics (PZT) with $3.0 \times 3.0 \times 0.2 \mathrm{~mm}$, i.e., one for excitation and the other for detection, are bonded on the vibrator.

On the other hand, a three-dimensional micro actuator, where a doubly L-shaped clamped-free beam type vibrator (see also Fig. 1) is utilized, is newly developed to enforce dynamic stimulations. Furthermore, incorporating the clamped-free micro sensor mentioned above into this three-dimensional actuator, we have newly constructed a three-dimensional actuator-sensor complex system, which enable us to have stimulation and detection onto the living cell almost simultaneously. It has three piezoelectric ceramics (sizes are the same as in the above sensor) in $\mathrm{x}, \mathrm{y}$ and $\mathrm{z}$ direction respectively and is able to perform three-dimensional dynamic actuation up to around $\pm 8 \mu \mathrm{m}$ in each direction. Figure 1(b) shows the developed three-dimensional actuator and sensor complex. In order to remove the ill effects of electrical interference like mutual inductance and to enhance the sensing capability, electrical wires, which are connected to each piezoelectric ceramics were fixed through a sponge block. At the tip of the sensor's vibrator, a nylon micro probe with a conically tapered end was bonded so that the induced mechanical stresses of the minute
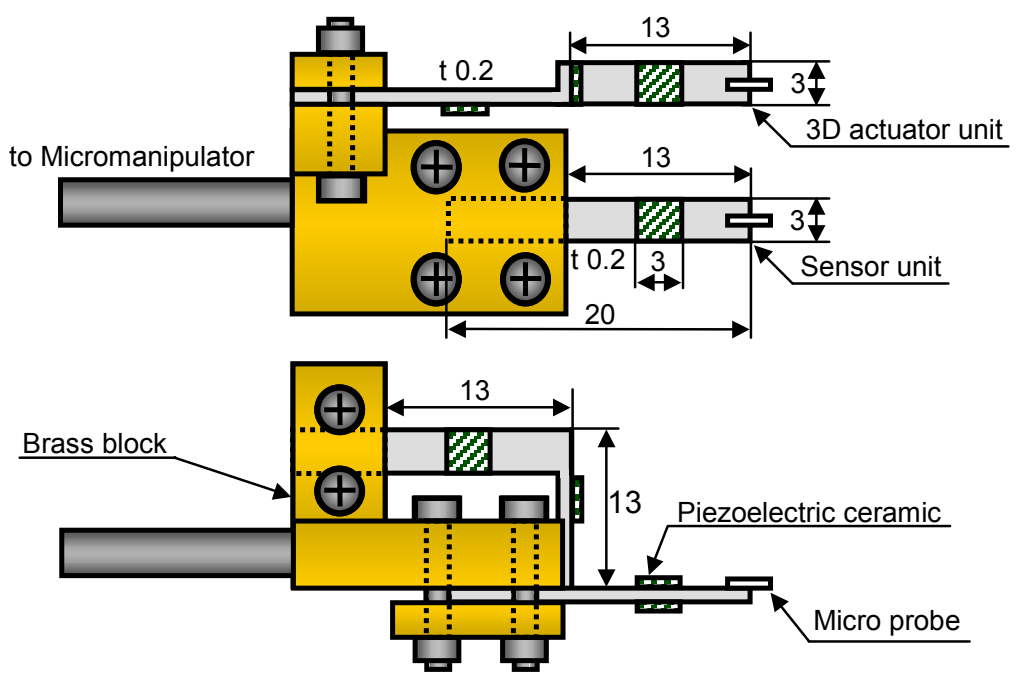

(a) Construction

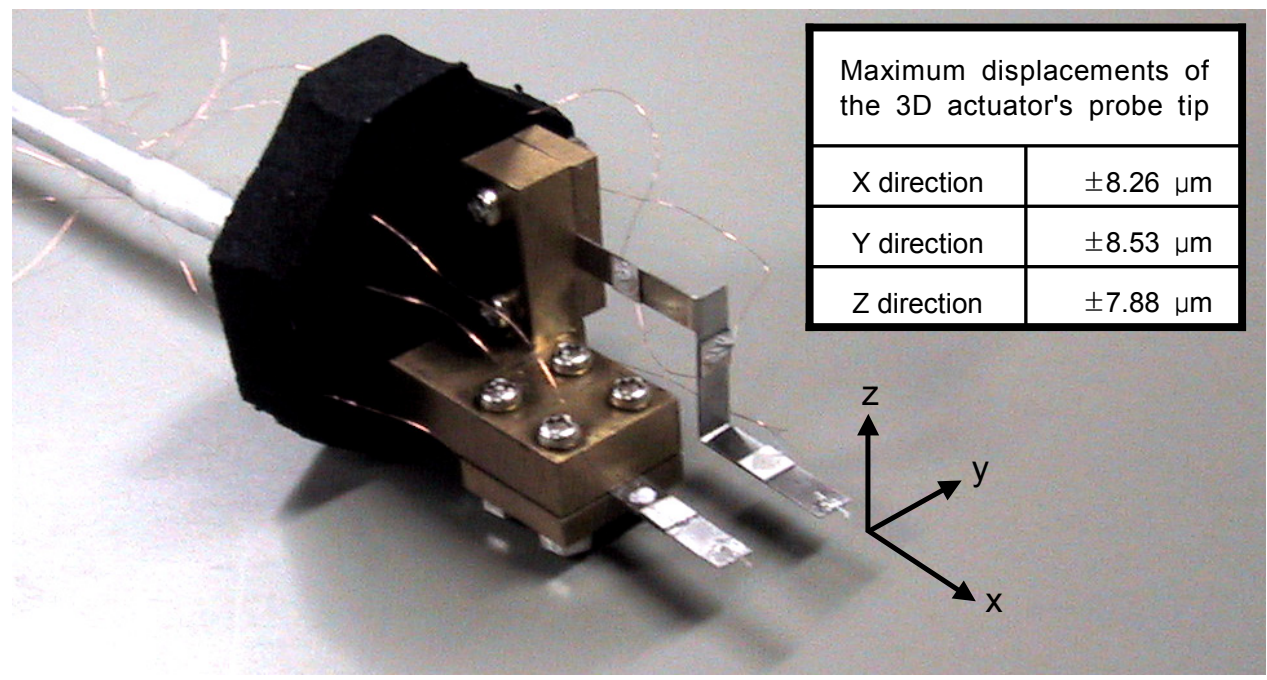

(b) Close up of the real 3D actuator-sensor complex and the actuator's maximum displacements

Fig. $13 \mathrm{D}$ actuator-sensor complex. 
cells and tissues were easily detected (Fig. 2). On the other hand, the actuator's tip has also a nylon micro probe of hemispherical end so that the dynamic stimulations can efficiently be enforced without causing membrane surface damage.

Although the experimental setup used for the present study is basically same as previous study ${ }^{(8)}$, it is briefly explained here for ease of understanding in the following experimental procedures (Fig. 3(a),(b)) and the discussion. The setup mainly consists of an electrically controlled micromanipulator system and cell injector, an inverted phase-contrast microscope system with CCD camera, a frequency generator, an amplifier and a dual channel FFT analyzer. The united three-dimensional actuator and sensor complex mentioned above is mounted on one side arm of a micromanipulator system. In the experiments, a micropipette connected with an electrically controlled cell injector, and a flow chamber connected with a constant-temperature water bath, are utilized. The sensor probe is brought up to the surface of the object. From the contact point, the probe is automatically maneuvered with $1 \mu \mathrm{m}$ step. In each case, one cycle of a swept sine wave is generated and after amplified appropriately, it is impulsively applied to the piezoelectric ceramic to excite the vibrator. Then the dynamic response of the vibrator is picked out and the natural frequency of the vibrator is given from power spectrum analysis by the FFT analyzer.

\subsection{Materials}

In this study, killifish's fertilized egg cells were selected as test subjects. Killifish is an endangered species having high sensitivity for chemical environments ${ }^{(9)(10)}$. It is suitable for genetic studies, since its heterogenesis is short such as 3-4 months. Moreover, structural changes in the initial activation process of the egg cells are easily observed by a light microscope. The spawning of killifish can be induced in any season by artificially controlled temperatures of $25-28^{\circ} \mathrm{C}$ with lighting cycles of 14 hours of light and 10 hours of dark. Once a female killifish enters into the breeding season, it spawns at dawn everyday. After fertilization, the egg cells are carefully picked out from the belly of the female killifish and are immersed in shallow water in a separate container with temperatures of $25-28^{\circ} \mathrm{C}$. The incubation period to hatch is strongly dependent on the temperatures and is about two weeks at the above mentioned temperature. The egg cell is captured by the micropipette, which is connected to one arm of the micromanipulator system. The aspiration pressure is electrically controlled by the cell injector. By this time, heart beat and blood circulation are easily observed under an optical microscope, and that makes possible to monitor the health condition of the egg cell during the experiment. As shown in Fig. 2, the sensor's micro probe is brought up to contact with the cell surface. From the contact point, the sensor probe is automatically indented with $1 \mu \mathrm{m}$ step toward the center of the egg cell.

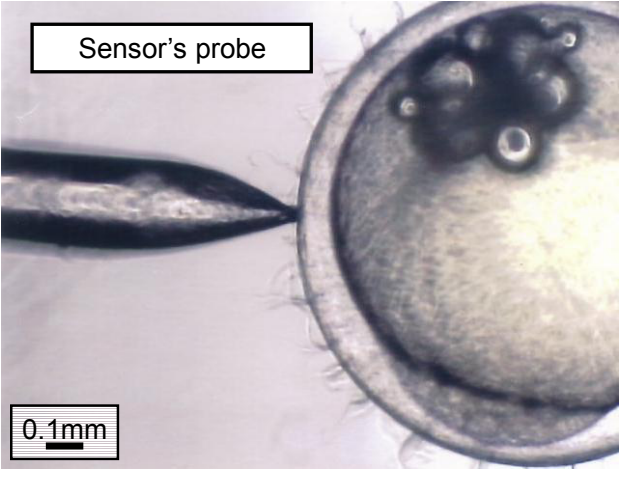

(a)

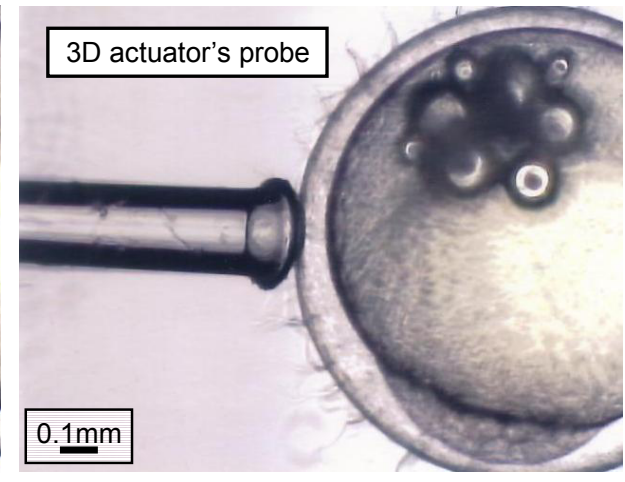

(b)

Fig. 2 Killifish's fertilized egg cell and two types of micro probe. 


\subsection{Validation experiments using silicone rubber blocks}

In this study, at first, four $50.0 \times 10.0 \times 3.0 \mathrm{~mm}$ silicone rubber blocks (manufactured by Koken Co., Ltd.) were experimentally used to see the capability of the sensor system. The softness was measured by the standard Shore hardness test ${ }^{(11)(12)}$. As shown in the schematic diagram of Fig. 3(a), the three-dimensional actuator-sensor complex is mounted on the left side arm of the micromanipulator system. Instead of the cell, the silicone rubber block is fixed on a glass strip mounted on the stage of the microscope system. The sensor probe is perpendicularly brought up to the surface of the silicone rubber block. From the contact point, the sensor is automatically maneuvered with $1 \mu \mathrm{m}$ step up to total displacement of $10 \mu \mathrm{m}$. In each case, one cycle of a swept sine wave is generated and after appropriate amplification, it is impulsively applied to the piezoelectric ceramics to excite the vibrator. Then the dynamic response of the vibrator is picked out and the natural frequency of the vibrator is given from power spectrum analysis by the FFT analyzer.

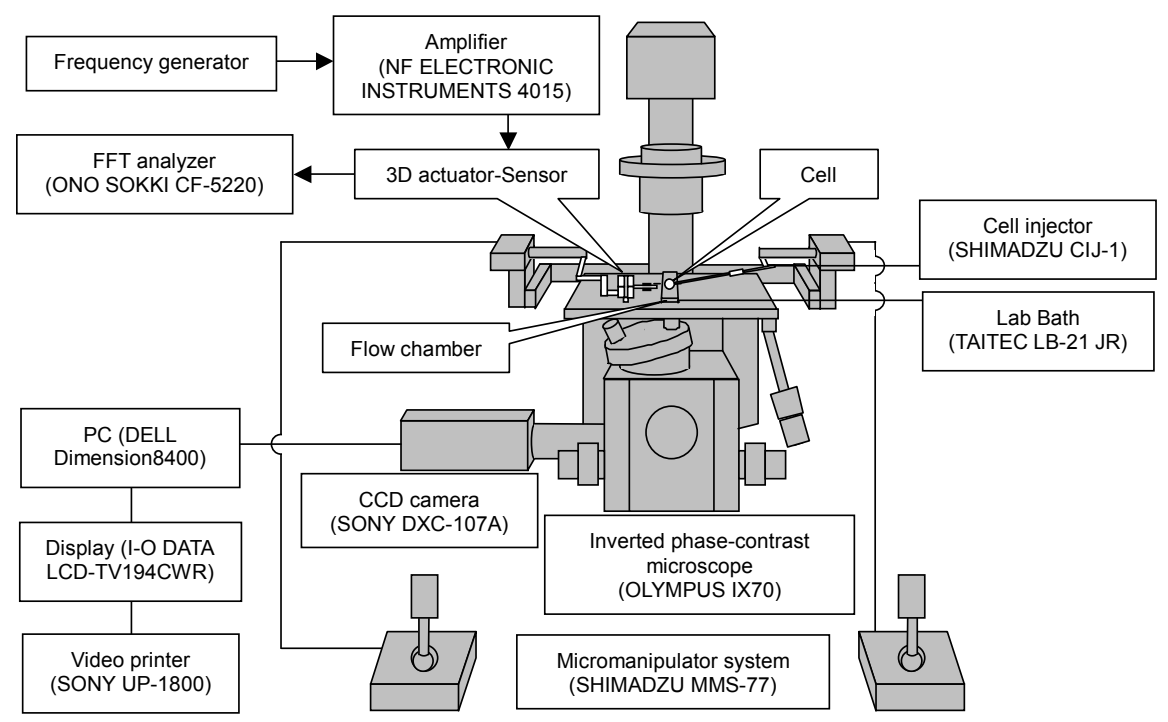

(a) Schematic diagram

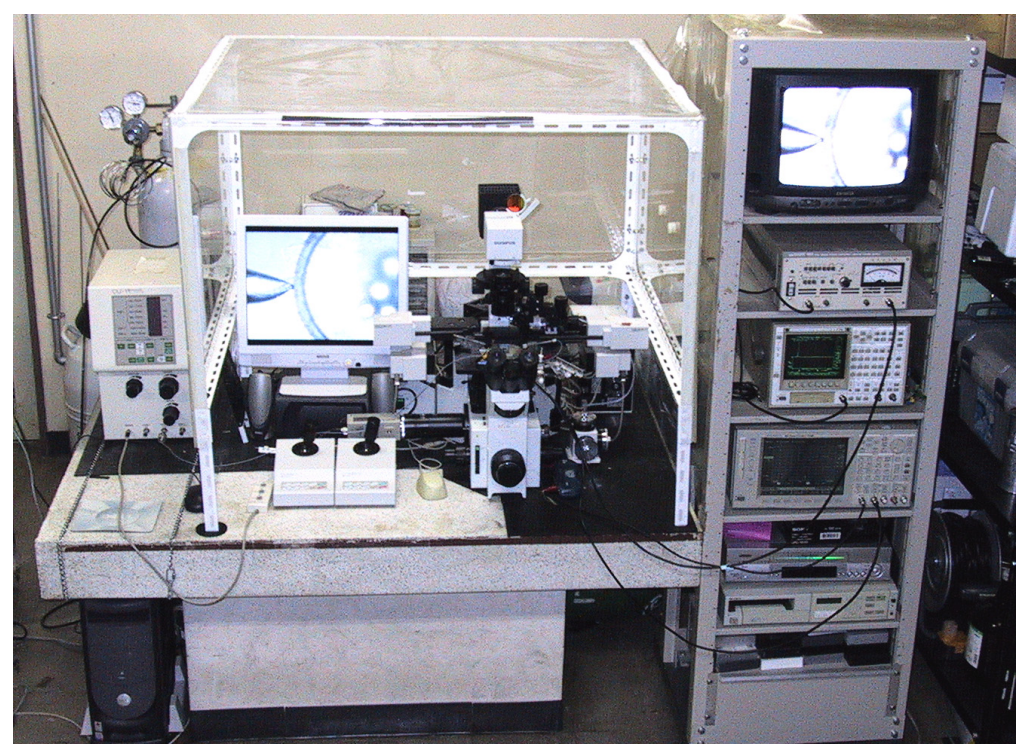

(b) Overall view

Fig. 3 Experimental setup 


\subsection{Experimental methods for killifish's fertilized egg cell}

As a first example, physiological responses of fertilized egg cells of killifish to environmental stresses induced by Glufosinate were examined. The Glufosinate is a main substance of commonly used weed killer and is suspected as a kind of environmental hormone. In this study, a Glufosinate Standard $(99.1 \%$, chemical name: Ammonium DL-Homoalanin-4-yl-(methyl)phosphinate) was provided by Wako Pure Chemical Industries, Ltd., and was diluted with distilled water (Otsuka Pharmaceutical Co., Ltd.) to the specific concentration, such as $100 \mathrm{ppm}$ or $10 \mathrm{ppm}$.

The egg cell is suspended in temperature controlled pure water on a glass strip of a specially designed flow chamber ${ }^{(7)}$ which is mounted on the stage of the inverted phase-contrast microscope system. The flow chamber consists of upper and lower glass strips and its four sides are covered with acrylic plates. A bank with two to three millimeter high is bonded on the top of the upper glass strip in order to prevent overflowing of the solution in which the cell is immersed. In the center part of the chamber, laminated square glass strips are inserted between the upper and lower glass strips in order to prevent defocusing by flowing water. Furthermore, two partition walls are set inside the chamber so as to guide the flow of water efficiently. Thermostated water is allowed to flow inside the chamber for temperature control. The egg cell is captured by the micropipette, which is connected to the right arm of the micromanipulator system. The aspiration pressure is electrically controlled by the cell injector. As shown in the schematic diagram of Fig. 3(a), the three-dimensional actuator-sensor complex is mounted on the left side arm of the micromanipulator system. With a constant probe indentation of $3 \mu \mathrm{m}$, the egg cell response was measured, while the Glufosinate solution was injected, so that the specific concentration settled down precisely to $100 \mathrm{ppm}$ or $10 \mathrm{ppm}$.

Secondly, mechanical reactions of the cells against dynamic stimulations were investigated. Stress responses were measured after 5 minutes of vibrating stimulations of 5 to $20 \mathrm{~Hz}$ with $15 \mu \mathrm{m}$ amplitude. The three-dimensional actuator's micro probe was indented with $30 \mu \mathrm{m}$. Here, two types of stimulations were applied, one is locally indenting vibration and the other is entirely cell swaying vibration. The sensor probe is constantly indented with $6 \mu \mathrm{m}$.

Finally, we examined the recovery progress of locally damaged cells by using the developed actuator-sensor complex system. The method, which induces damage on the cell, is similar to the general cell manipulation procedures utilized in the in-vitro

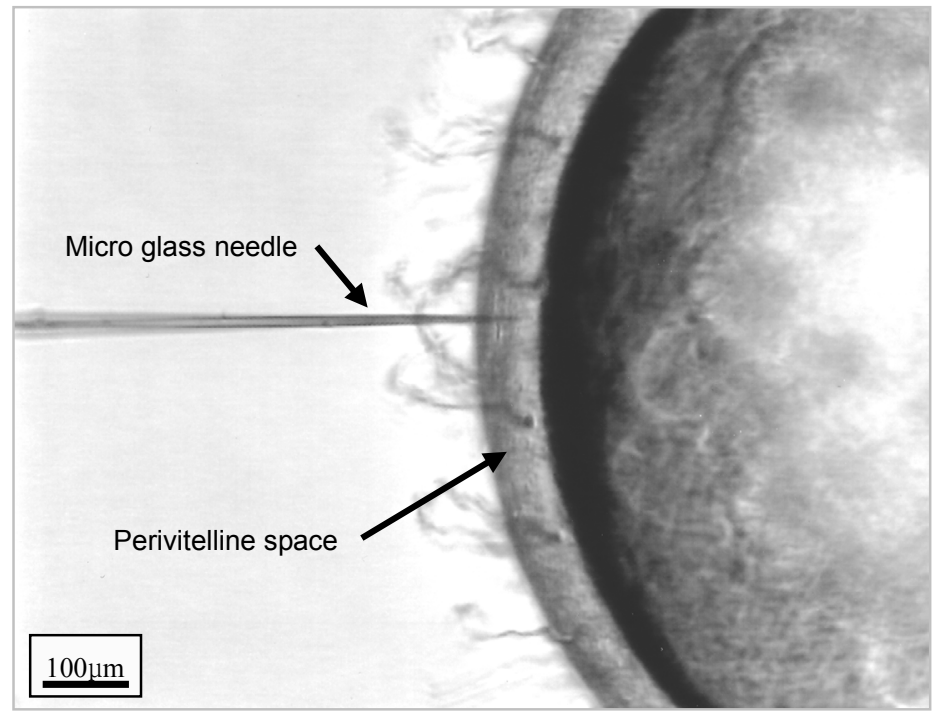

Fig. 4 Local damage to the fertilized egg cell by perforating with micro glass needle. 
fertilization technique by using micro glass needle (Fig. 4). The micro needle mounted on the left side arm of the micromanipulator system is perforated until three fourth $(70-80 \mu \mathrm{m})$ of the total thickness of the perivitelline space ${ }^{(13)}$ so that it does not punch through. Comparisons were made between the normal cells, the locally damaged cells and the dynamically stimulated cells after being locally damaged. Practical procedures for the locally damaged cell are schematically shown in Fig. 5. After the perforation, replacing the micro glass needle with the three-dimensional actuator-sensor complex, 5 minutes of vibrating stimulation $(20 \mathrm{~Hz}$ with $16 \mu \mathrm{m}$ amplitude) were enforced by the three-dimensional actuator's micro probe with constant indentation of $20 \mu \mathrm{m}$ onto the egg cell membrane. Immediately after the stimulation, the micro sensor of the complex was moved on the surface of the egg cells. Then a contact point was sought between the sensor probe and the cell membrane surface. The frequency parameter $\Delta \mathrm{f} / \mathrm{f}_{0}$ was measured with the sensor probe indentation at each $1 \mu \mathrm{m}$ step, up to $10 \mu \mathrm{m}$.

\section{Results and discussion}

Figure 6 shows the relationships between the sensor's normalized frequency parameter: $\Delta \mathrm{f} / \mathrm{f}_{0}$ and probe indentation for four different silicone rubber blocks. Here, $\Delta \mathrm{f}$ is the change in natural frequency before and after a contact of the probe, and $\mathrm{f}_{0}$ denotes the natural frequency for free boundary at the probe end. Frequency parameter increases significantly in the order of 20,40,60 and 80HS. Generally, it also increases as the probe indentation increases. The surface of the $80 \mathrm{HS}$ is fairly stiff and the steep curve in the figure represents this property. On the other hand, the surface of the $20 \mathrm{HS}$ is quite soft and the curve is almost flat without showing significant increases in frequency. These results suggest that each curve represents the characteristics of the material's compliance, which is not available only by the Shore hardness test. Thus, the sensing capability of this sensor system was well demonstrated.

In order to investigate the immediate egg cell response to the Glufosinate, Figure 7 shows the relationships between the sensor frequency parameter $\Delta \mathrm{f} / \mathrm{f}_{0}$ and the elapsed time after the Glufosinate injection into the surrounding solution (10 or $100 \mathrm{ppm})$ with a constant conical probe indentation of $3 \mu \mathrm{m}$. Each point represents the mean value of measured data. To avoid congestion in the figure, error bars were omitted. Green circles denote the results

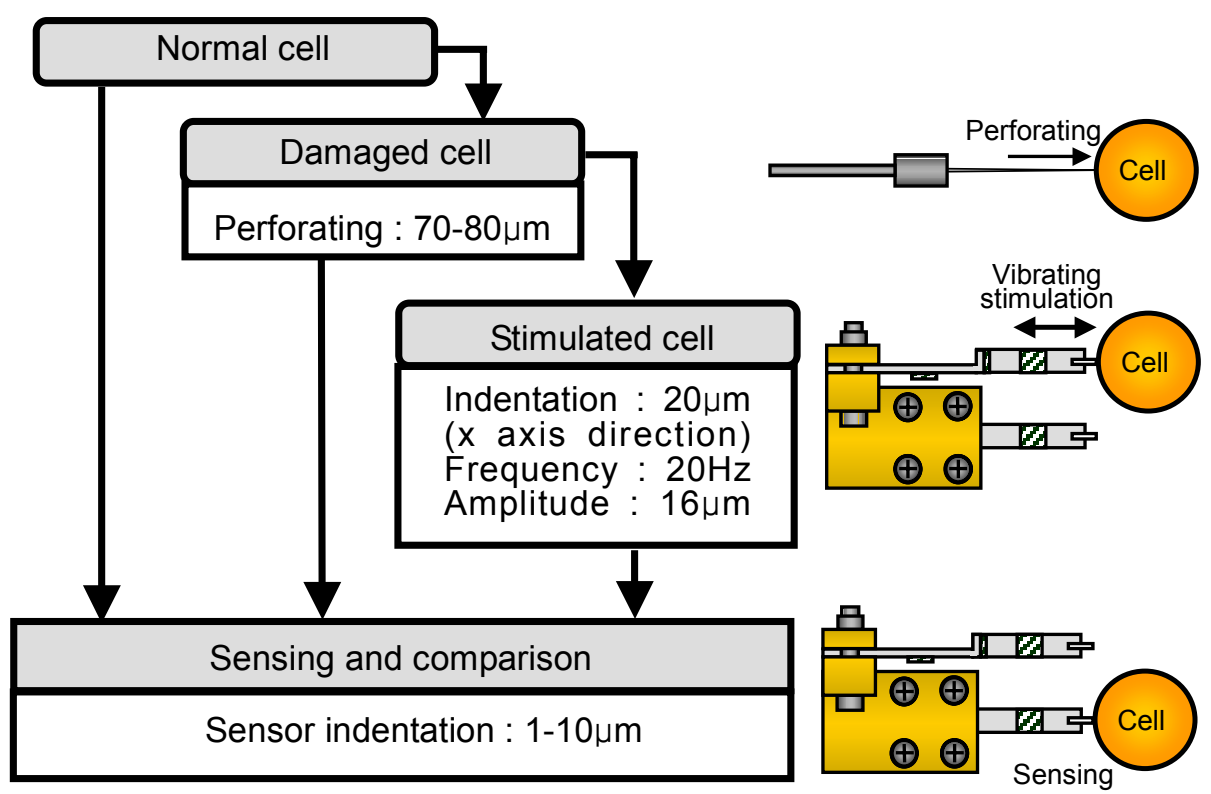

Fig. 5 Experimental procedures for the locally damaged cell. 
of egg cells submerged in pure water, while blue circles denote the results where the Glufosinate was injected during the time 0 to 4 seconds, until the concentration became $10 \mathrm{ppm}$. Red circles denote the result for $100 \mathrm{ppm}$ case. In pure water case, the result remains almost constant, while in the $10 \mathrm{ppm}$ case, the results are going down right after the Glufosinate injection and continue to do so until around 24 minutes, where they settle down

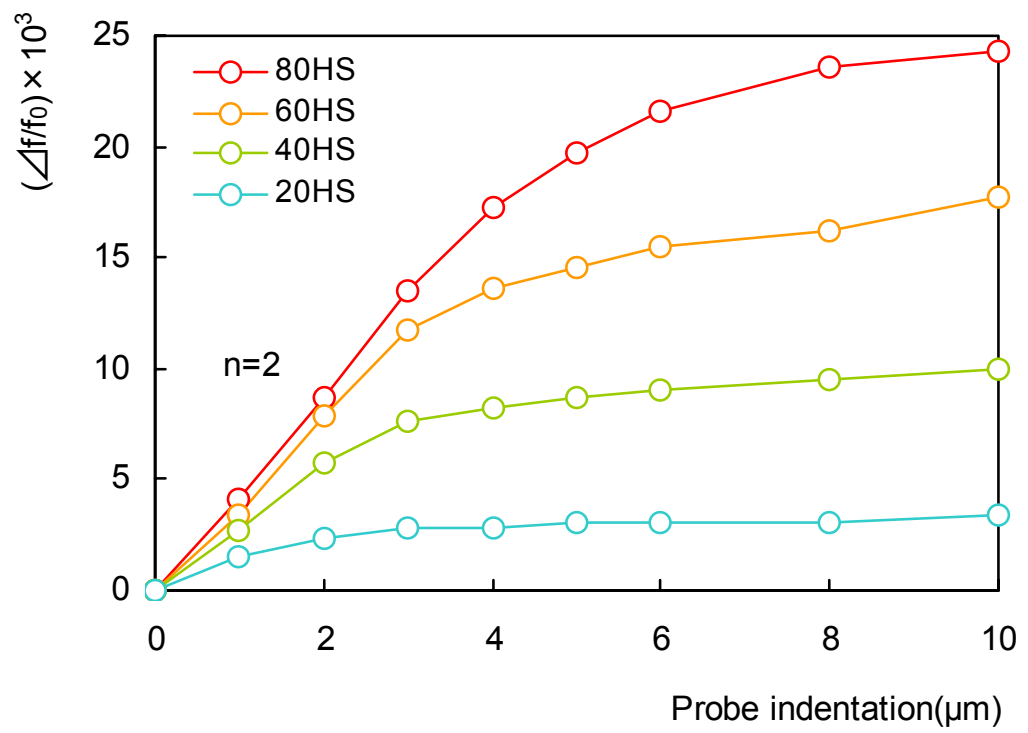

Fig. 6 Frequency responses of silicone rubber blocks. The frequency parameter increases significantly in the order of 20,40,60 and $80 \mathrm{HS}$. It also increases as the probe indentation increases. The sensing capability of this sensor system was well demonstrated.

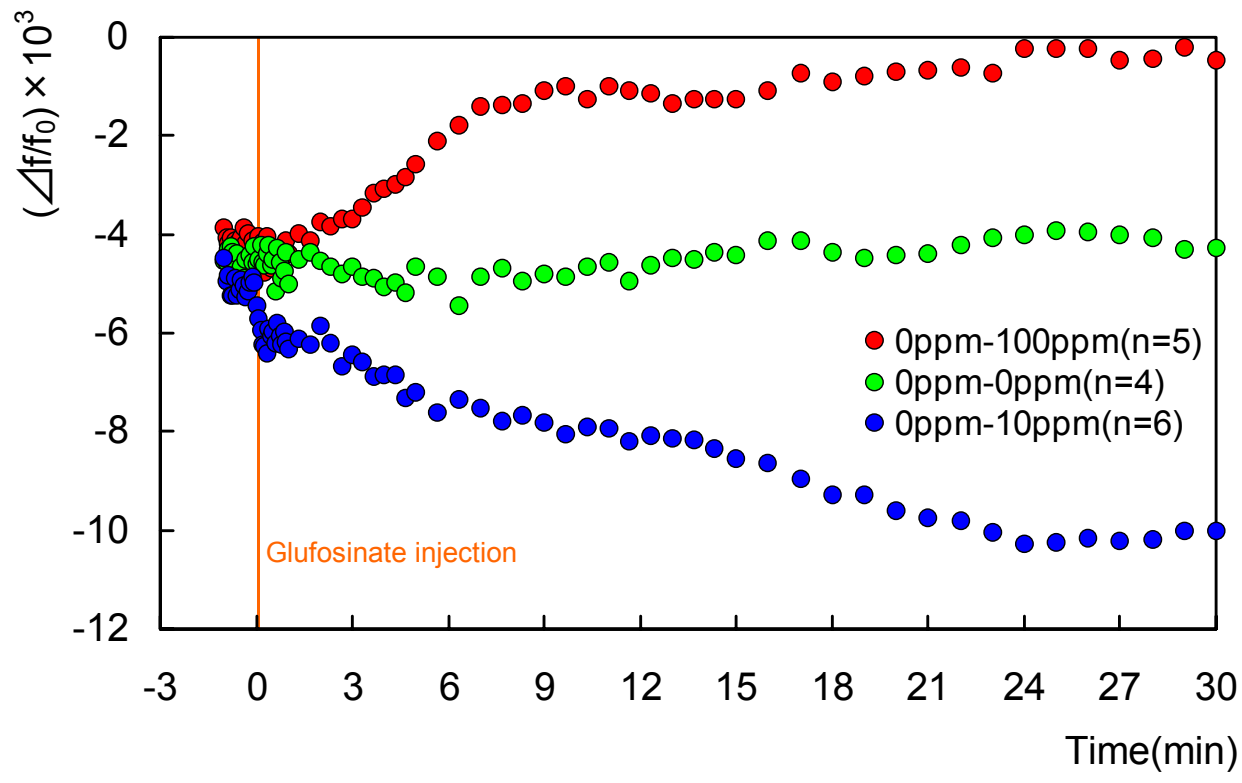

Fig. 7 Egg cell response to Glufosinate (main substance of typical weed killer), 2 days after fertilization. The probe indentation was constant with $3 \mu \mathrm{m}$. In the $100 \mathrm{ppm}$ case, the results are going up just after the Glufosinate injection due to acute toxicity, while in the $10 \mathrm{ppm}$ case the results are going down probably due to the "low concentration effect". These phenomena are considered to reflect the initial adaptation process of the cell to the surrounding environments. 
to constant. In the $100 \mathrm{ppm}$ case, the results are going up just after the Glufosinate injection and continue to do so until around 7 minutes, where they settle down to constant. The effect of acute toxicity of high concentration of the Glufosinate is considered to be a probable main reason for the above characteristics. That is, some protection mechanisms for cytoplasm, which prevent the cell to be softened, might be initiated in the plasma membrane. As for the $10 \mathrm{ppm}$ case, decreasing in the frequency parameter might be due to the so-called "low concentration effect" of the environmental hormone. The low concentration effect is a special function where specific substance works effectively at an ultra low concentration condition, such as hormones secreted in animals or environmental hormones. These phenomena might reflect the initial adaptation process of the cell to the surrounding environments. Thus monitoring for the environmental stresses induced in living cells may be realized with the aid of the present sensor system.

Response to dynamic stimulations upon killifish's fertilized egg cell is shown in Fig. 8. In this figure, $\mathrm{f}_{0}$ is the value of the sensor's frequency before the dynamic stimulation is imposed. The sensor's probe is constantly indented $6 \mu \mathrm{m}$ into the egg cell membrane surface. $\Delta \mathrm{f}$ denotes the value of sensor's frequency changed from $\mathrm{f}_{0}$ after the dynamic stimulation is imposed. Here, two types of stimulations were applied, one is locally indenting vibration and the other is entirely cell swaying vibration. In the indenting vibration case, all results are going down according to time. It seems that the egg cells were initially strongly stressed by local stimulation, then relaxation of the stress is occurring with time. It is observed that the response to dynamic stimulations might have frequency dependence. On the other hand, in the swaying vibration case, it is interestingly found that

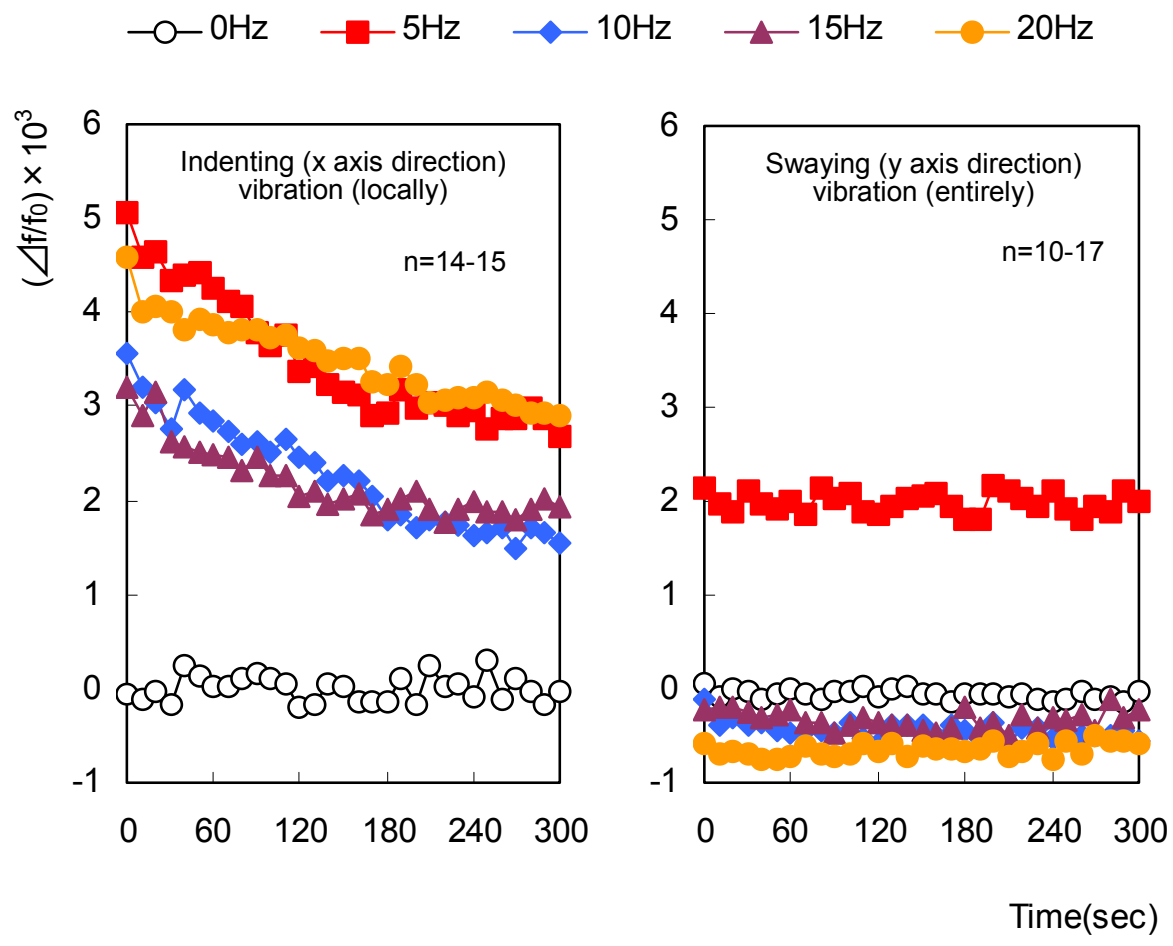

Fig. 8 Mechanical reactions of killifish's egg cell against dynamic stimulations (6hrs after fertilization). Stress responses were measured after 5 minutes of vibrating stimulations of 5 to $20 \mathrm{~Hz}$ with $15 \mu \mathrm{m}$ amplitude. The actuator's micro probe was initially indented with $30 \mu \mathrm{m}$ and the sensor's probe is constantly indented with $6 \mu \mathrm{m}$. Two types of stimulations were applied, one is locally indenting vibration and the other is entirely cell swaying vibration. It is observed that the response to dynamic stimulation depends on frequency. 
there is a large reaction to $5 \mathrm{~Hz}$ stimulation. This special reaction might be connected with killifish's heart beat frequency.

As the final example, influences of local damage and dynamic stimulation upon killifish's fertilized egg cell were examined. In this case, dynamic stimulations were imposed at $20 \mathrm{~Hz}$ indenting vibration, which were notable in the experiment shown in Fig. 8. Both the local damage and dynamic stimulation were administered at 6 hours after fertilization. The results are shown in Fig. 9. Figure 9(a) shows the result right after the perforation treatments mentioned above in Fig. 4. It is found that surface membrane of the damaged egg cell is harder than in the normal cell. Furthermore, the membrane of the stimulated egg cell is harder than in the damaged cell. These phenomena are considered that surface membrane of the egg cell becomes harder due to the initiation of a defense mechanism of the cell itself in response to artificial local damage or dynamic stimulation. It is also found that the results of the dynamically stimulated case are scattering, and that indicates specificity of the cell is large against the dynamic stimulation. Figure 9(b) shows the results of 24 hours later for the same egg cells of Fig. 9(a). The tendencies are almost same as those in Fig. 9(a). In this figure, however, when the sensor indentation is larger than $3 \mu \mathrm{m}$, the normal cell becomes softer than in Fig. 9(a). The reason for the temporal softening phenomenon at this time is considered so-called rhythmical contractile movement ${ }^{(9)}$ of initial developmental stages of the egg cell of killifish. This movement causes instability of the protoplasmic membrane and consequently leads to decrease of surface tension as mentioned in discussion of Fig. 10 of Ref. (7). Figure 9(c) shows the result of 48 hours later for the same egg cells of Fig. 9(a). At this stage, significant differences are not seen among the normal cells, the locally damaged cells and the

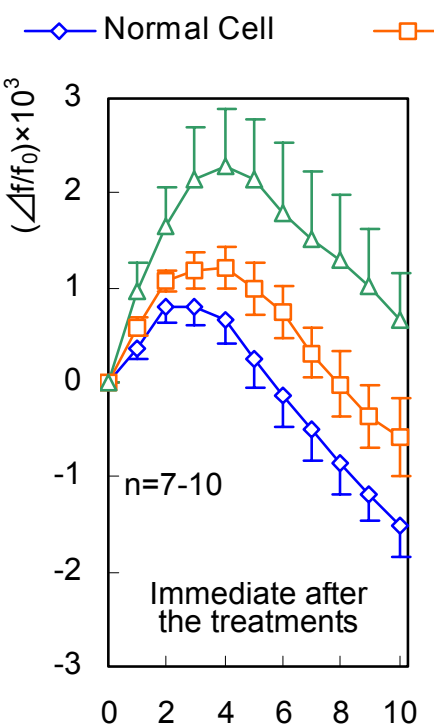

(a)

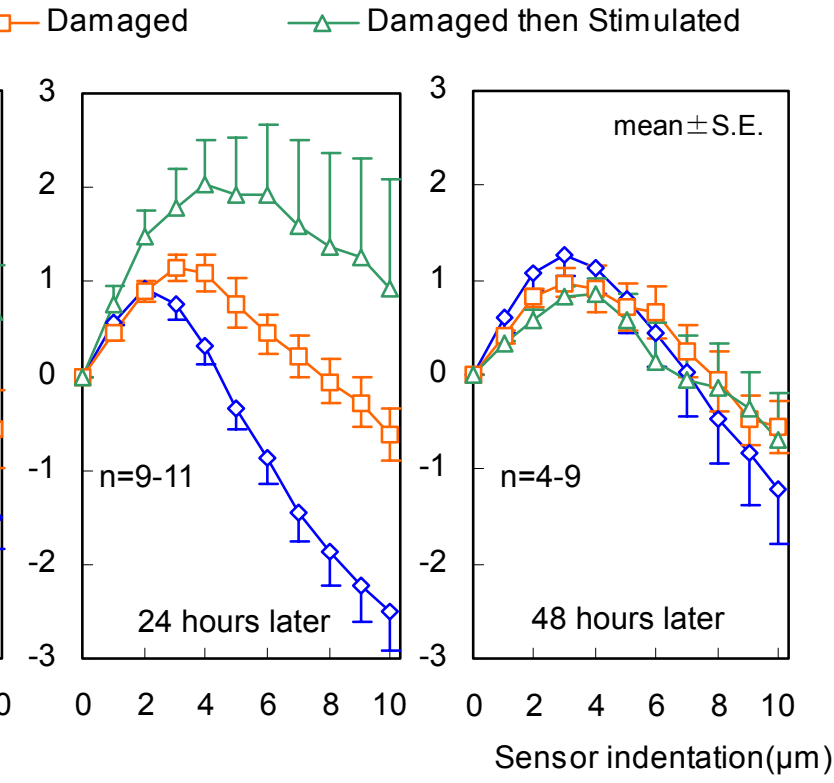

(b)

(c)

Fig. 9 Influences of local damage by micro glass needle and dynamic stimulation upon killifish's egg cell (constant probe indentation with $20 \mu \mathrm{m}, 5 \mathrm{~min}$ of $\pm 8 \mu \mathrm{m}$ amplitude indenting vibration with $20 \mathrm{~Hz}$ ) (6hrs after fertilization). It is found that surface membrane of the damaged egg cell is harder than the normal cell, while the stimulated egg cell is harder than the damaged cell. Both the damaged cell and the dynamically stimulated cell are considered to be normalized within 48 hours or so. 
dynamically stimulated cells. Also, the specificity seen among the dynamically stimulated cells can not be seen clearly in Fig. 9(c). Consequently, it is observed that state of both the damaged cell and dynamically stimulated cell might be normalized within a time span of 48 hours or so.

It is well known that the killifish egg cell's chorion continues to become harder ${ }^{(10)}$ just after fertilization for the purpose of self defense. It is considered to reflect this tendency that the increasing characteristics with respect to time are clearly seen in its hardness of the normal cells except for the case where the sensor indentation is larger than $3 \mu \mathrm{m}$ in Fig. 9(b). On the other hand, the damaged cells become hard immediate after the damage, presumably for defense purpose, and seems to continue to keep its state within the time span of 48 hours, though the physiological reason of this phenomenon is not yet clear.

Omata $^{(4)}$ has developed a micro tactile sensor to detect softness of cells using longitudinal vibration of a rod shaped piezoelectric element. In his study, the stiffness of internal organs, such as the intestine, the integument, the ovary, the heart, in an earthworm was examined utilizing a micro metal needle tactile sensor with $5 \mu \mathrm{m}$ in diameter. Validity of the method has been demonstrated by conducting data calibration using gelatin. However, comparable data regarding to living cells is not available in the study. In the present study, a new noninvasive micro three-dimensional actuator-sensor system is developed not only to detect compliance of minute living cells but also to impose dynamic stimulations upon living cells. Calibration of the data can systematically be obtained based on the experiment utilizing silicone rubber blocks whose Shore hardness is known.

It is commonly pointed out that there exists some sort of correlation between the recovering activities of living cells and tissues from local damage and externally imposed dynamic stimulation. The present study has been carried out to make contributions to this field. Sizes of the egg cells used in this study are of order $1 \mathrm{~mm}$, while the sizes of human cells are around ten to hundred micron meters. By making a few adjustments in shapes and sizes of the micro probe, the present method can be extended in sensing and actuating these human living cells. Since the present three-dimensional actuator-sensor complex is designed to be lightweight and compact, it is not difficult to be installed in conventional microscopes and micromanipulator systems. Furthermore, the actuator-sensor system has the feasibility to be used even in biologically clean environments in $\mathrm{CO}_{2}$ cell incubators, since it is easy to be sterilized due to its simply designed structure and compactness.

\section{Conclusion}

In this study, a new noninvasive micro three-dimensional actuator-sensor system is developed not only to detect compliance of minute living cells but also to impose dynamic stimulations upon living cells.

1. A three-dimensional micro actuator has been developed to enforce dynamic stimulations onto minute living cell.

2. Experimental studies have shown the sensor's capability to detect changes of the environmental stresses induced in egg cells of killifish.

3. The method has shown that the reaction of living cells against vibrating stimulations by the actuator might have frequency dependence.

4. The recovering process of locally damaged egg cells was clearly observed by the present system.

\section{Acknowledgements}

The authors wish to thank Messrs. Tadashi Rokugawa and Naoki Endo for their contributions in the experiments and data processing. The authors also wish to thank Dr. 
Mikael Langthjem, associate professor of Yamagata University, for his useful suggestions in revision of the manuscript. This work was supported in part by research grants from JSPS Grant-in-Aid for Scientific Research, Nos. 14655089 and (B) 15360119.

\section{References}

(1) Alberts, B., Johnson, A., Lewis, J., Raff, M., Roberts, K. and Walter, P., Molecular biology of the cell, 4th ed., Garland Science, New York (2002), pp. 583-1027.

(2) Mow, V.C., Guilak, F., Tran-Son-Tay, R. and Hochmuth, R.H., (eds) Cell mechanics and cellular engineering, Springer, Berlin Heidelberg New York (1994).

(3) Atala, A. and Lanza, R.P., Methods of tissue engineering, Academic Press (2002).

(4) Omata, S., Development of micro tactile sensor for detecting stiffness of cell using piezoelectric element and applications, Technical digest of the 14th sensor symposium (1996), pp. 203-206.

(5) Kosawada, T. and Kuni-i, K., A novel method of sensing for "Softness" by using dynamic response of piezoelectric vibrator, Proceedings of the international conference on new frontiers in biomechanical engineering, Tokyo (1997), pp. 429-432.

(6) Kosawada, T., Kuni-i, K. and Arikawa, K., Mechanical stresses induced in cell and their sensing by using dynamic response of piezoelectric micro-vibrator, Proceedings of the 4th international conference on motion and vibration control, Zurich, 1 (1998), pp. 183-188.

(7) Kosawada, T., Sato, H. and Nishida, H., Development of a softness sensing system by using dynamic response of piezoelectric vibrator and its application to detect mechanical properties of living cells, JSME International Journal C43(4) (2000), pp. 772-779.

(8) Kosawada, T., Konno, K., Yamamura, S. and Kanazawa, T., Piezo micro probe sensor system to detect environmental stresses induced in living cells and tissues, Microsystem Technologies, 11(2005), pp. 943-949.

(9) Yamamoto, T., Medaka (Killifish) Biology and strains, Keigaku Pub. Co., (1975), pp. 59-72.

(10) Iwamatsu, T., Changes of the Chorion upon fertilization in the Medaka, Oryzias latipes, The bulletin of Aichi University of Education 18 (1969), pp. 43-56.

(11) Uchikoshi, T., Mechanical Materials, Tokyo Denki University Press (in Japanese) (1987), pp 20-25.

(12) Shore hardness test - Test method, JIS Z 2246-2000 (in Japanese).

(13) Yamamoto, T., Medaka (Killifish) Biology and strains, Keigaku Pub. Co., (1975), pp. $30-44$. 